\title{
The role of mixed vibronic Qy-Qx bands in the light- harvesting dynamics of the major antenna complex, LHCII
}

\section{Eric Arsenault}

University of California, Berkeley https://orcid.org/0000-0002-5363-3229

\section{Yusuke Yoneda}

University of California, Berkeley https://orcid.org/0000-0003-4974-6842

\section{Masakazu Iwai}

Lawrence Berkeley National Laboratory https://orcid.org/0000-0002-0986-9015

\section{Krishna Niyogi}

Howard Hughes Medical Institute, University of California https://orcid.org/0000-0001-7229-2071

Graham Fleming ( $\square$ grfleming@lbl.gov)

University of California, Berkeley https://orcid.org/0000-0003-0847-1838

\section{Article}

Keywords: photosynthesis, electronic-nuclear mixing, $\mathrm{Chl} \mathrm{a,} \mathrm{Chl} \mathrm{b,} \mathrm{light-harvesting}$

Posted Date: August 4th, 2020

DOl: https://doi.org/10.21203/rs.3.rs-46465/v1

License: (c) (i) This work is licensed under a Creative Commons Attribution 4.0 International License. Read Full License

Version of Record: A version of this preprint was published at Nature Communications on November 26th, 2020. See the published version at https://doi.org/10.1038/s41467-020-19800-y. 
The role of mixed vibronic $Q_{y}-Q_{x}$ bands in the light-harvesting dynamics of the major antenna complex, LHCII

Eric A. Arsenault ${ }^{1,2,3}$, Yusuke Yoneda ${ }^{1,2,3}$, Masakazu Iwai ${ }^{3,4}$, Krishna K. Niyogi ${ }^{3,4}$, and Graham R. Fleming ${ }^{1,2,3^{*}}$

${ }^{1}$ Department of Chemistry, University of California, Berkeley, CA 94720, USA

${ }^{2}$ Kavli Energy Nanoscience Institute at Berkeley, Berkeley, CA 94720, USA

${ }^{3}$ Molecular Biophysics and Integrated Bioimaging Division, Lawrence Berkeley National Laboratory, Berkeley, CA 94720, USA

${ }^{4}$ Department of Plant and Microbial Biology, University of California, Berkeley, CA 94720, USA "grfleming@1bl.gov

The importance of green light for driving natural photosynthesis has long been underappreciated, however, under the presence of strong illumination, green light actually drives photosynthesis more efficiently than red light. This green light is absorbed by mixed vibronic $Q_{y}-Q_{x}$ states, arising from chlorophyll $(\mathrm{Chl})$-Chl interactions, although almost nothing is known about these states. Here, we employ polarization-dependent twodimensional electronic-vibrational spectroscopy to study the origin and dynamics of the mixed vibronic $Q_{y}-Q_{x}$ states of light-harvesting complex II. We show the states in this region dominantly arise from $\mathrm{Chl} b$ and demonstrate how it is possible to distinguish between the degree of vibronic $Q_{y}$ versus $Q_{x}$ character. We find that the dynamics for states of predominately $C h l b Q_{\mathrm{y}}$ versus $C h l b Q_{\mathrm{x}}$ character are markedly different, as excitation persists for significantly longer in the $Q_{x}$ states and there is an oscillatory component to the $Q_{x}$ dynamics, which will be discussed. Our findings demonstrate the central role of electronic-nuclear mixing in efficient light-harvesting and the different functionalities of $\mathrm{Chl}$ $a$ and Chl $b$. 


\section{Introduction}

The fact that leaves are green and the majority of spectroscopic studies on optically active pigment-protein complexes (PPCs) are performed on in vitro systems has led to the misunderstanding that green light has little efficacy on photosynthesis. However, on the contrary, it has been shown by Terashima et al. that for in vivo systems, green light in the presence of strong illumination actually has the ability to drive photosynthesis more efficiently than red light. ${ }^{1}$ In this work, we explore the states absorbing green light and their dynamics in light-harvesting complex II (LHCII).

Overall, the success of the photosynthetic apparatus begins with the design and function of the PPCs which harvest solar light, the primary step in photosynthesis. ${ }^{2}$ In green plants and algae, LHCII serves as the major antenna complex, which transfers excitation energy towards the photosynthetic reaction center. ${ }^{2}$ LHCII, in trimeric form as it is generally found, is composed of 24 chlorophyll (Chl) $a, 18 \mathrm{Chl} b$, and 12 carotenoid (Car) pigments. ${ }^{3}$ Spatially, these pigments are held within the protein environment in such a way that electrostatic interactions between nearby pigments promote the formation of delocalized excitonic states, leading to intricately tuned spatial and energetic landscapes. Together, these pigments, preferentially arranged over billions of years of evolution $^{4,5}$, harvest light with a quantum efficiency of near unity. ${ }^{2}$

Understanding how the excitation energy transfer (EET) dynamics of LHCII are mapped across the spatial and energetic degrees of freedom (DoF) of the complex has been the focus of significant, sustained attention. ${ }^{6-22}$ This effort has increasingly led to a deeper understanding of Chl-Chl interactions, which predominately manifest energetically in the red edge of the LHCII absorption spectrum (excitonic Qy bands of mainly Chl $a$ and Chl $b$ character give rise to the two peaks centered around $14800 \mathrm{~cm}^{-1}$ and $15500 \mathrm{~cm}^{-1}$ in Figure 1a, respectively). ${ }^{14}$ Recent work has 
also more closely considered the role of Car-Chl interactions, which arise largely at higher energies, i.e. at the blue edge of the LHCII absorption spectrum (peak shoulder rising around 19000 $\mathrm{cm}^{-1}$ in Figure 1a). ${ }^{17,18}$ However, over $3000 \mathrm{~cm}^{-1}$ of the spectrum, namely the Chl vibronic $Q_{y}-Q_{x}$ region energetically connecting the Chl $\mathrm{Q}_{\mathrm{y}}$ states and the Car states ${ }^{23}$, remains little studied. This is not particularly surprising, despite the estimated integrated absorption of the vibronic $\mathrm{Q}_{\mathrm{y}}-\mathrm{Q}_{\mathrm{x}}$ region being nearly 75\% of that of the $\mathrm{Q}_{\mathrm{y}}$ region in LHCII, because these states have low oscillator strength and are highly mixed in multiple ways, thus making them challenging to study. Namely, the states of LHCII that span this spectral region have varying degrees of i) mixed inter-pigment electronic character, ii) mixed intra-pigment electronic character $\left(\mathrm{Q}_{\mathrm{x}} / \mathrm{Q}_{\mathrm{y}}\right.$ character $)$, and iii) vibronic character (mixed electronic-vibrational states). The understated importance of the

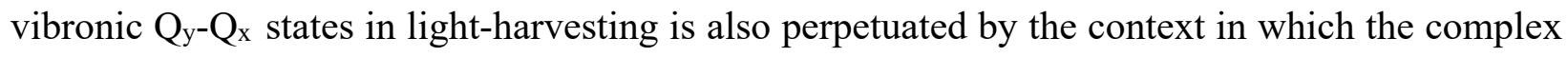
is typically studied - in vitro - rather than in vivo. In fact, previous work has shown that absorption of the vibronic $\mathrm{Q}_{\mathrm{y}}-\mathrm{Q}_{\mathrm{x}}$ states of $\mathrm{Chl}$ is enhanced in leaves relative to isolated LHCII, as well as other minor PPCs, to such an extent that the spectrum is essentially uniform over the full photosynthetically active region (PAR). ${ }^{24-27}$ This phenomenon, termed the detour effect, is caused by the highly light scattering environment within the leaf which effectively increases the optical path length of incident green light, therefore, increasing the likelihood of absorption. ${ }^{25,27}$ The result is that such a significant increase in the light-harvesting contribution of the vibronic $\mathrm{Q}_{\mathrm{y}}-\mathrm{Q}_{\mathrm{x}}$ region occurs such that these states rival the contribution from the Qy electronic region. ${ }^{1,24}$ Through an analogous mechanism, yet on even larger scale, green light is also crucial in stimulating photosynthesis in the lower portions of the canopy where red and blue light have been filtered out by the top layers. ${ }^{27}$ It is worth noting here that this comparison is in terms of quantum efficiency rather than overall energy efficiency. ${ }^{28,29}$ This is to say that the additional energy over the $\mathrm{Q}_{\mathrm{y}}$ region 
contained by the photons absorbed by the vibronic $Q_{y}-Q_{x}$ region is unable to be utilized by photosynthesis because the energy required to drive charge separation in the photosynthetic reaction center is equivalent to that of a red, rather than green, photon. ${ }^{29}$ However, in vivo, green light drives photosynthesis more successfully in chloroplasts throughout the leaf and plant (despite diminished energy efficiency). This is because of the improved quantum efficiency of the vibronic $\mathrm{Q}_{\mathrm{y}-\mathrm{Q}} \mathrm{x}$ states due to the detour effect and the fact that the photons absorbed by these states ultimately penetrate deeper into the mesophyll of a given leaf and the canopy of a whole plant than do the red photons, which are more readily absorbed by the $\mathrm{Q}_{\mathrm{y}}$ electronic states arising from PPCs residing nearer to the surface of the leaf. However, the lack of spectral assignments or insight into the EET dynamics of the Chl vibronic $\mathrm{Q}_{\mathrm{y}}-\mathrm{Q}_{\mathrm{x}}$ states has long hindered a complete understanding of their in role photosynthetic light-harvesting.

In this work, we utilize recent advances in multidimensional spectroscopy, namely the advent of two-dimensional electronic-vibrational (2DEV) spectroscopy ${ }^{30}$, to study the origin and involvement of these highly mixed states - inaccessible to more conventional spectroscopies.

A major advantage of this technique is the improved spectral resolution, afforded by IR detection, which has successfully allowed for insight into ultrafast energy transfer, charge transfer, and proton transfer dynamics. ${ }^{6,19,31-34}$ IR detection also inherently makes this technique especially sensitive to the mixing of vibronic states because such mixing significantly alters vibrational transitions moments. Additionally, the further sensitivity provided by polarization-dependent 2DEV spectroscopy has been demonstrated in the spectral assignments of monomeric $\mathrm{Chl} a$ and $b^{35}$, as well as in unveiling the role of vibronic coupling in a solar cell dye. ${ }^{34}$

Here, building on previous applications of this technique, particularly to LHCII ${ }^{6,19}$ and monomeric $\mathrm{Chl} a$ and $\mathrm{Chl} b^{35}$, we apply polarization-dependent 2DEV spectroscopy to study the 
origin of the highest-lying mixed vibronic $\mathrm{Q}_{y}-\mathrm{Q}_{\mathrm{x}}$ states (spanning 520-570 $\mathrm{nm}$ ) arising from ChlChl interactions in LHCII, in order to gain mechanistic insight into their function in photosynthetic light-harvesting. In doing this, we present direct evidence that this spectral region is dominated by Chl $b$ character, which together with previous in vivo studies indicates that $\mathrm{Chl} b$ enhances the ability of green plants and algae to harvest green light. ${ }^{1,24-26}$ Following more definitive assignments, we will discuss the role of these states in the EET dynamics of LHCII at $77 \mathrm{~K}$. Namely, we show that relaxation from the higher-lying states of mainly $\mathrm{Chl} b$ character to the lower-lying $\mathrm{Q}_{\mathrm{y}}$ states occurs on a timescale of $<90 \mathrm{fs}$ (within our instrument response function), demonstrating how mixing between the electronic and nuclear DoF of Chl $b$ drives the ultrafast EET dynamics of LHCII and extends efficient light-harvesting throughout the PAR. Further, we find that relaxation from the $\mathrm{Q}_{\mathrm{x}} \rightarrow \mathrm{Q}_{\mathrm{y}}$ states of $\mathrm{Chl} b$ occurs on a timescale of $\sim 200 \mathrm{fs}$ (based on the timescales of an oscillatory component associated directly with these states). Such a timescale for $\mathrm{Q}_{\mathrm{x}} \rightarrow \mathrm{Q}_{\mathrm{y}}$ transfer in Chl $b$ agrees well with recent theoretical work on monomeric $\mathrm{Chl} b^{36}$, which suggests that the observed $\mathrm{Q}_{\mathrm{x}}$ states arise from more highly localized $\mathrm{Chl} b$ pigments. The ability of polarization-dependent $2 \mathrm{DEV}$ to follow the pathways of energy flow for such highly mixed states of LHCII offers a new direction towards a deeper understanding of photosynthetic lightharvesting across the solar spectrum.

\section{Results and Discussion}

2DEV spectroscopy, a two-color multidimensional spectroscopic experiment, features visible pump pulses that prepare an ensemble of electronic/vibronic states that evolve during the waiting time, $T$, and are tracked via an IR probe pulse. ${ }^{30}$ The data is presented in the form of excitation frequency-detection frequency correlation plots that map how the electronic/vibronic states evolve with considerable frequency resolution - made possible by IR detection. 
The 2DEV measurements were performed in two different polarization schemes - one in which the visible pump pair and IR probe were all vertically polarized, $S_{V}\left(\omega_{\text {exc. }}, T, \omega_{\text {det. }}\right)$, and one in which the visible pump pair was horizontally polarized while the IR probe was vertically polarized, $S_{H}\left(\omega_{\text {exc. }}, T, \omega_{\text {det. }}\right)$. These were combined to generate the perpendicular and parallel polarization-associated (PA) 2DEV spectra, given by: ${ }^{37,38}$

$$
S_{\perp}\left(\omega_{\text {exc. }}, T, \omega_{\text {det. }}\right)=3 S_{H}\left(\omega_{\text {exc. }}, T, \omega_{\text {det. }}\right)-S_{V}\left(\omega_{\text {exc. }}, T, \omega_{\text {det. }}\right)
$$

and

$$
S_{\|}\left(\omega_{\text {exc. }}, T, \omega_{\text {det. }}\right)=2 S_{V}\left(\omega_{\text {exc. }}, T, \omega_{\text {det. }}\right)-S_{H}\left(\omega_{\text {exc. }}, T, \omega_{\text {det. }}\right)
$$

respectively. The perpendicular or parallel distinction indicates the angle between the electronic transition dipole moment (TDM) of states initially populated by the visible pump pair and the vibrational TDM of the probed mode on the states populated during the waiting time, $T$, that will be amplified in the respective PA spectra. As will be shown below, PA 2DEV spectra are particularly useful for separating the evolution of different states (e.g. states of $\mathrm{Q}_{\mathrm{x}}$ or $\mathrm{Q}_{\mathrm{y}}$ character) in highly congested, complex spectra.

Along with the linear absorption spectrum of LHCII (Fig. 1a), Figure 1 shows a representative 2DEV spectrum obtained via exciting the $\mathrm{Q}_{\mathrm{y}}$ bands $\left(14350-16775 \mathrm{~cm}^{-1}\right)$ of LHCII (Figure 1b), discussed in detail in previous work ${ }^{6,19}$, along with PA 2DEV spectra of a portion of the vibronic $Q_{y}-Q_{x}$ region (17545-19230 $\mathrm{cm}^{-1}$ ) of the LHCII spectrum (Figure 1c-f). In the 2DEV spectrum of the $\mathrm{Q}_{\mathrm{y}}$ bands of LHCII at $T=130 \mathrm{fs}$, multiple distinct bands along the excitation axis 


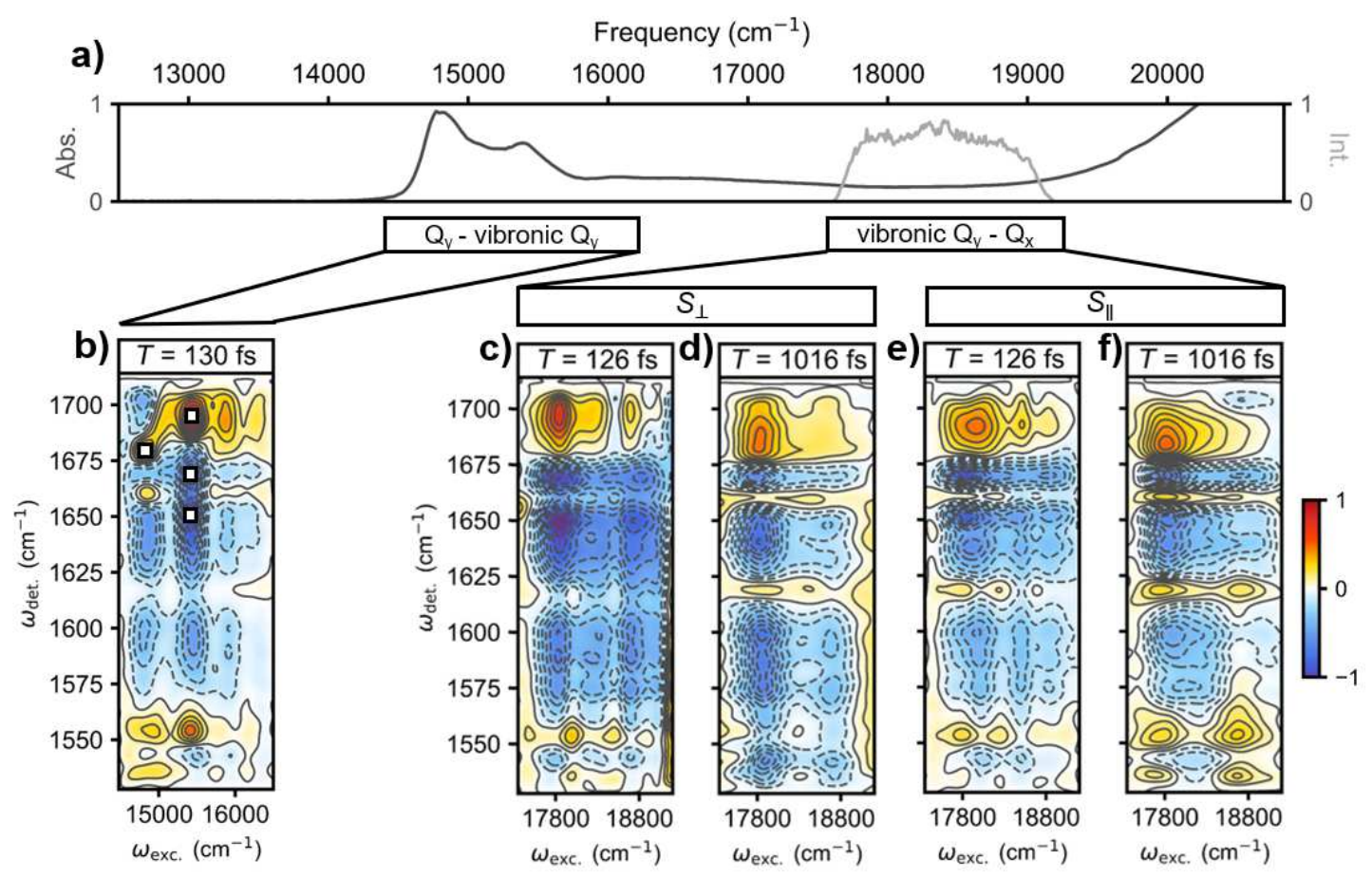

Figure 1. Linear absorption and two-dimensional electronic-vibrational spectra of LHCII. a) Linear absorption spectrum of LHCII at $77 \mathrm{~K}$, along with the spectrum of the visible pump pulses employed in this work (light gray). b) Representative 2DEV spectrum of the $\mathrm{Q}_{\mathrm{y}}$ band of LHCII at $T=130 \mathrm{fs}$, prior to significant Chl $b \rightarrow \mathrm{Chl} a$ transfer. Throughout, ground state bleach (GSB) features are positive (red) features and excited state absorption (ESA) features are negative (blue) features. Four regions have been marked in the spectra with white squares - one GSB in the predominately Chl $a$ excitonic band (centered around $14800 \mathrm{~cm}^{-1}$ ) and one GSB and two ESA in the predominately Chl $b$ excitonic band (centered around $15500 \mathrm{~cm}^{-1}$ ). 2c)-d) Perpendicular PA 2DEV spectra of the vibronic $\mathrm{Q}_{\mathrm{y}}-\mathrm{Q}_{\mathrm{x}}$ bands of LHCII at $77 \mathrm{~K}$ at waiting times of $T=126 \mathrm{fs}$ and $1016 \mathrm{fs}$. e)-f) Parallel PA 2DEV spectra of the vibronic $\mathrm{Q}_{\mathrm{y}}-\mathrm{Q}_{\mathrm{x}}$ bands of LHCII at $77 \mathrm{~K}$ at waiting times of $T=126 \mathrm{fs}$ and $1016 \mathrm{fs}$.

are evident. Bands centered around $14800 \mathrm{~cm}^{-1}$ and $15500 \mathrm{~cm}^{-1}$ correspond to the excitonic states of mainly Chl $a$ and $\mathrm{Chl} b$ character, respectively, while the bands above $15600 \mathrm{~cm}^{-1}$ correspond to higher-lying vibronic $\mathrm{Q}_{\mathrm{y}}$ transitions originating from mainly $\mathrm{Chl} b .{ }^{6,19} \mathrm{It}$ is clear from comparing the bands of mainly $\mathrm{Chl} a$ and $\mathrm{Chl} b$ character at $T=130 \mathrm{fs}$, prior to significant $\mathrm{Chl} b \rightarrow \mathrm{Chl} a$ transfer, that there are differences in the vibrational structure, as particular modes have predominately $\mathrm{Chl} a$ or $b$ character.

Four distinct spectral regions that have been marked in Figure $1 \mathrm{~b}$ in order to assign and track the dynamics of the vibronic $Q_{y}-Q_{x}$ region. Of the four detection frequencies of interest, two are ground state bleach (GSB) features (red, positive features), at $1680 \mathrm{~cm}^{-1}$ and $1690 \mathrm{~cm}^{-1}$, 
assigned to Chl $a$ and $\mathrm{Chl} b$, respectively, and two are excited state absorption (ESA) features (blue, negative features). These two ESA features, at $1650 \mathrm{~cm}^{-1}$ and $1670 \mathrm{~cm}^{-1}$, are notably dominant in the Chl $b$ band. Previously, the ESA around $1650 \mathrm{~cm}^{-1}$ was assigned to have predominately $\mathrm{Chl} b$ character from more localized chromophores, supported by a singular value decomposition analysis that revealed that this region of the spectrum decayed on a timescale of a few picoseconds, a characteristic timescale of transfer from more localized $\mathrm{Chl} b$ intermediate states to Chl $a .^{6}$ Although an assignment for the feature at $1670 \mathrm{~cm}^{-1}$ was not totally conclusive, the spectral regions around $1650 \mathrm{~cm}^{-1}$ and $1670 \mathrm{~cm}^{-1}$ evolved on similar timescales and it is clearly a dominant ESA in the band of mainly $\mathrm{Chl} b$ origin, suggesting that the $1670 \mathrm{~cm}^{-1}$ band also has significant character from more localized $\mathrm{Chl} b$ states.

A comparison of the predominantly $\mathrm{Chl} a$ and $\mathrm{Chl} b$ bands in the $\mathrm{Q}_{\mathrm{y}}$ region (Figure 1b) versus the PA 2DEV spectra in the vibronic $\mathrm{Q}_{\mathrm{y}}-\mathrm{Q}_{\mathrm{x}}$ region at $T=126 \mathrm{fs}$ (Figure $1 \mathrm{c}$ and e) shows that across the spectrum, detection frequencies assigned either completely or predominantly to $\mathrm{Chl}$ $b$ - the GSB at $1690 \mathrm{~cm}^{-1}$ and ESAs at $1650 \mathrm{~cm}^{-1}$ and $1670 \mathrm{~cm}^{-1}$ - dominate the spectral structure along the detection axis. After about a picosecond (Figure 1d and f), there is noticeable decay of these three peaks, as a peak at $1680 \mathrm{~cm}^{-1}$, the GSB of Chl $a$, grows in. Immediately, the dominance of Chl $b$ excited states in this region of the LHCII spectrum is clearly demonstrated.

In order to gain a more quantitative understanding of the composition of region of the LHCII absorption spectrum (Chl $a$ versus $\mathrm{Chl} b$ character and $\mathrm{Q}_{\mathrm{y}}$ versus $\mathrm{Q}_{\mathrm{x}}$ character), it is helpful to view the PA 2DEV spectra in a different way. The best visualization of the dynamics is in the form of a plot of excitation frequency versus waiting time at a fixed detection frequency (Figure $2 \mathrm{a}, \mathrm{b}, \mathrm{d}$, and e). To construct these plots, slices through the $2 \mathrm{D}$ spectra will be taken at a fixed detection frequency along the excitation axis and plotted as a function of the waiting time. The 
benefit of this visualization is that if the detection frequency is fixed on a local ground state mode, as will be done here, then this serves as an anchor point to assess the relative orientation between the TDM of this vibrational mode and the TDM of the populated excited state(s). Specifically, to assess the character of this region, the GSB detection frequencies will be set to $1690 \mathrm{~cm}^{-1}$ (Figure 2a and b) and $1680 \mathrm{~cm}^{-1}$ (Figure $2 \mathrm{~d}$ and e), to track $\mathrm{Chl} a$ and $\mathrm{Chl} b$ character, respectively. Based on the 2DEV anisotropy data of monomeric $\mathrm{Chl} b$, the angle between the $\mathrm{Q}_{\mathrm{y}}$ and $\mathrm{Chl} b$-specific GSB (related to the formyl group specific to $\mathrm{Chl} b$ ) TDMs is approximately $60^{\circ} .{ }^{35}$ Therefore, as the $\mathrm{Q}_{\mathrm{y}}$ and $\mathrm{Q}_{\mathrm{x}} \mathrm{TDMs}$ are approximately orthogonal ${ }^{39}$, the angle between the $\mathrm{Q}_{\mathrm{x}}$ and $\mathrm{Chl} b$ GSB should be approximately $30^{\circ}$. When the probed mode is fixed on the Chl $b$ GSB, the $S_{\|}$spectra will selectively amplify the Qx pathway, while suppressing the Qy and vice versa for the $S_{\perp}$ spectra. Within this framework, the significant differences between the perpendicular and parallel PA spectra can be explained in a relatively straightforward way - each of these spectra is mainly capturing the evolution of different excited states. This is of course a simplification of the scenario in practice, as the pigments are embedded in a protein environment and are surrounded by and interacting with neighboring pigments, all of which can influence the TDMs, however, not to the extreme to which the Qy orientation should rotate so significantly that it would be amplified in $S_{\|}$, at least for this fixed detection frequency. The analysis here will focus on the region centered around $17800 \mathrm{~cm}^{-1}$ (Figure 2; highlighted by red and blue boxes in $S_{\perp}$ and $S_{\|}$, respectively), but much of what is found here can be easily extrapolated to the higher frequency around $18700 \mathrm{~cm}^{-1}$ (Figure 2; highlighted by green boxes). Monitoring the peak amplitude of the Chl $b$ GSB evolution around $17800 \mathrm{~cm}^{-1}$ reveals the immediate appearance of this feature in both $S_{\perp}$ and $S_{\|}$. This is shown more explicitly in Figure 2c, which shows the evolution of the Chl $b$ GSB peak amplitude at an excitation frequency of $17800 \mathrm{~cm}^{-1}$. The initial amplitudes of the Chl $b$ GSB indicates that 


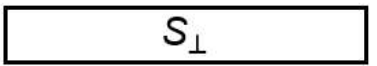

a) $\omega_{\text {det. }}=1690 \mathrm{~cm}^{-1}$

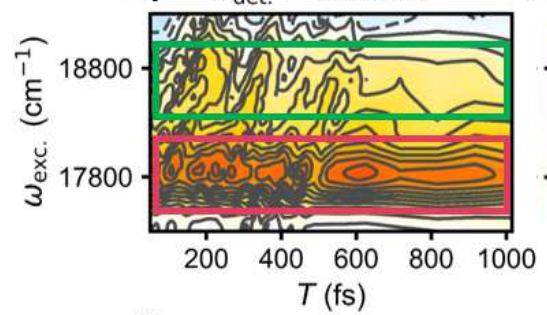

b)
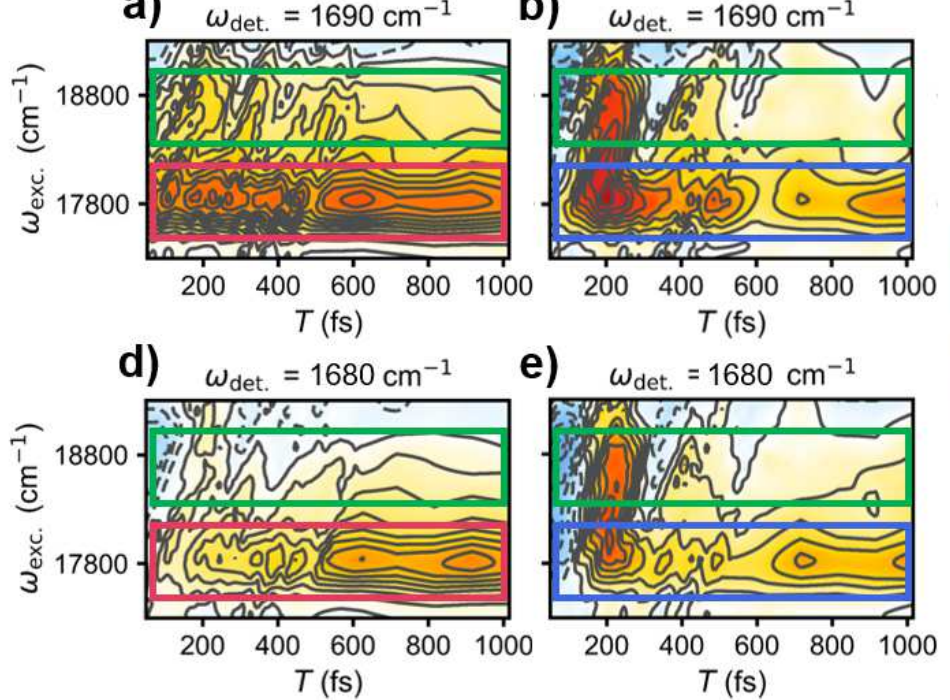

e)

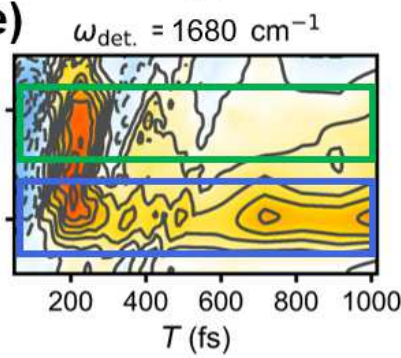

c)
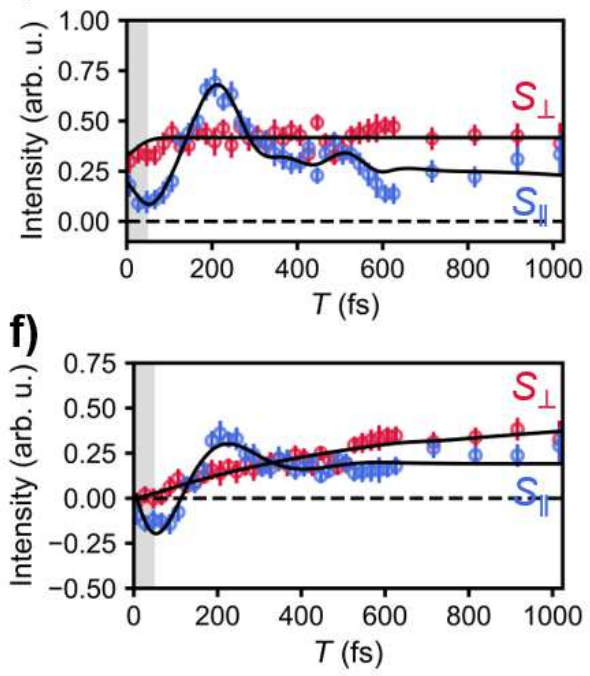

Figure 2. Ground state bleach dynamics along the excitation axis. a)-b) Perpendicular and parallel PA spectral evolution as a function of waiting time along the excitation axis at a fixed detection frequency of $1690 \mathrm{~cm}^{-1}$ (indicative of Chl $b$ population). Ground state bleach features are positive (red) features and excited state absorption features are negative (blue) features. The frequency region centered around $17800 \mathrm{~cm}^{-1}$ is boxed in red for the perpendicular PA component $\left(S_{\perp}\right)$ and blue for the parallel PA component $\left(S_{\|}\right)$. Throughout, green boxes indicate the higher frequency region centered around $18700 \mathrm{~cm}^{-1}$. c) Peak amplitude dynamics at a detection frequency of $1690 \mathrm{~cm}^{-1}$ along 17800 $\mathrm{cm}^{-1}$ (through the center of the corresponding boxed regions of the same color). Error bars indicate the estimated errors in the peak amplitude based on the standard deviation of the signal amplitude at each waiting time. The black lines indicate the fits of the peak amplitude dynamics (provided in Supplementary Table 1). The gray shaded region indicates the region where pulse overlap effects occur. d)-e) Same as described for a)-b) except at a detection frequency of $1680 \mathrm{~cm}^{-1}$ (indicative of $\mathrm{Chl} a$ population). f) Same as c) except at a detection frequency of $1680 \mathrm{~cm}^{-1}$.

this region has both substantial Chl $b$ Q character (selectively amplified in $S_{\|}$) and Chl $b$ vibronic Qy character (selectively amplified in $S_{\perp}$ ). A comparison of the spectra around $17800 \mathrm{~cm}^{-1}$ versus $18700 \mathrm{~cm}^{-1}$, shows that the higher frequency region still has vibronic $\mathrm{Q}_{\mathrm{y}} \mathrm{Chl} b$ character, although it is weaker (Figure 2a; region highlighted in red box versus green box), as well as significant $\mathrm{Q}_{\mathrm{x}}$ character, as there is only a slight reduction between the relative signal strengths (Figure 2b; region highlighted in blue box versus green box). To confirm the dominance of $\mathrm{Chl} b$, analysis of the spectral evolution at the Chl $a$ GSB detection frequency, $1680 \mathrm{~cm}^{-1}$, reveals that in both the $S_{\|}$and $S_{\perp}$ spectra (Figure $2 \mathrm{~d}$ and e), there is essentially no Chl $a$ character initially. This is more clearly demonstrated in Figure $2 \mathrm{f}$ which shows that both the parallel and perpendicular PA components 
of the Chl $a$ GSB peak amplitude along $17800 \mathrm{~cm}^{-1}$ have initial intensities of zero. In addition, a clear rise can be observed in the perpendicular component with a timescale of $600 \pm 200 \mathrm{fs}$, which agrees well with other previously observed $\mathrm{Chl} b \rightarrow \mathrm{Chl} a$ transfer timescales. ${ }^{9,16,20}$ A more indepth analysis of the parallel component for this feature of Chl $a$ is difficult as there is a degree of spectral congestion even with IR detection. For example, there is an overlapping negative oscillatory ESA signal in this same detection region clearly visible in the higher frequency region in Figure 2e. Unfortunately, this overlap makes it difficult to determine if there is some small amount of Chl $a$ character in this region or if there is some rapid Chl $b \rightarrow \mathrm{Chl} a$ transfer in less than 200 fs. The clearly observed oscillatory behavior in the parallel component of the 2DEV spectra will be discussed below.

Figure 3a, b, d, and e present the evolution of the excited state frequency distributions along the excitation axis as a function of waiting time at fixed detection frequencies of $1670 \mathrm{~cm}^{-1}$ and $1650 \mathrm{~cm}^{-1}$. The focus of the ESA analysis will be at a detection frequency of $1670 \mathrm{~cm}^{-1}$, rather than at $1650 \mathrm{~cm}^{-1}$, because there is less spectral congestion in this region, although the evolution between at these two detection frequencies is nearly identical (Figure $3 a$ and $d$ versus Figure $3 b$ and e). We note that this is further evidence that these two ESAs have similar origins, i.e. a large degree of character from $\mathrm{Chl} b$ pigments only weakly coupled to neighboring chromophores. For the analysis, focus will be on the spectral region around $17800 \mathrm{~cm}^{-1}$ because the energy levels in this region clearly participate more strongly in facilitating transfer to $\mathrm{Chl} a$ and therefore play a more significant role in the dynamics. For both of these ESAs, the parallel PA spectra have a significant oscillatory component, as was also seen for the GSB features, and is largely absent in the perpendicular PA spectra for all four detection frequencies. This suggests that the underlying origin of these features is the same, i.e. $S_{\|}$selectively isolates the $\mathrm{Q}_{\mathrm{x}}$ contribution of these ESA 
features. This may also indicate that these features in part arise from the formyl group specific to $\mathrm{Chl} b$, as then the TDM of these excited state vibrational modes should have a similar orientation to the ground state vibrational mode of $\mathrm{Chl} b$ (also is related to the formyl group). The attribution of Chl $b \mathrm{Q}_{\mathrm{x}}$ as the origin of this oscillatory signal is further supported by the fact that not only do the ESA and GSB both show oscillations in $S_{\|}$, but a fit of the ESA at $1670 \mathrm{~cm}^{-1}$ along $17800 \mathrm{~cm}^{-}$ ${ }^{1}$ (Figure 3c; shown in blue) recovers frequencies of $106 \pm 5 \mathrm{~cm}^{-1}$ and $240 \pm 10 \mathrm{~cm}^{-1}$, while a fit of the GSB recovers identical frequencies within error: $111 \pm 7 \mathrm{~cm}^{-1}$ and $240 \pm 20 \mathrm{~cm}^{-1}$ (error indicates $1 \sigma$ interval, see Supplementary Table 1 for complete fit results). The finding of identical frequencies strongly indicates that $S_{\|}$isolates the Qx component of the GSB and ESA detection frequencies, especially because no such dynamics are observed in any of the perpendicular PA. This striking oscillatory behavior is made even more interesting as it is observed in both ESA and GSB features.

Before discussing the origin of these oscillations, it is worth mentioning that the amplitude of the $1670 \mathrm{~cm}^{-1}$ ESA along $17800 \mathrm{~cm}^{-1}$ in $S_{\perp}$ undergoes a monoexponential decay on a timescale of $600 \pm 200$ fs (Figure 3c; shown in red), identical to the rise in the Chl $a$ GSB. Again, this timescale falls within the range of observed $\mathrm{Chl} b \rightarrow \mathrm{Chl} a$ transfer. However, there is clearly a longer timescale component (beyond the duration of the experiment) to the ESA signal because the peak amplitude has yet to fully decay by one picosecond. This is as expected because this feature likely has a significant degree of character from more localized Chl $b$ pigments which undergo $\mathrm{Chl} b \rightarrow \mathrm{Chl} a$ transfer the timescale of a few picoseconds. ${ }^{15}$ 

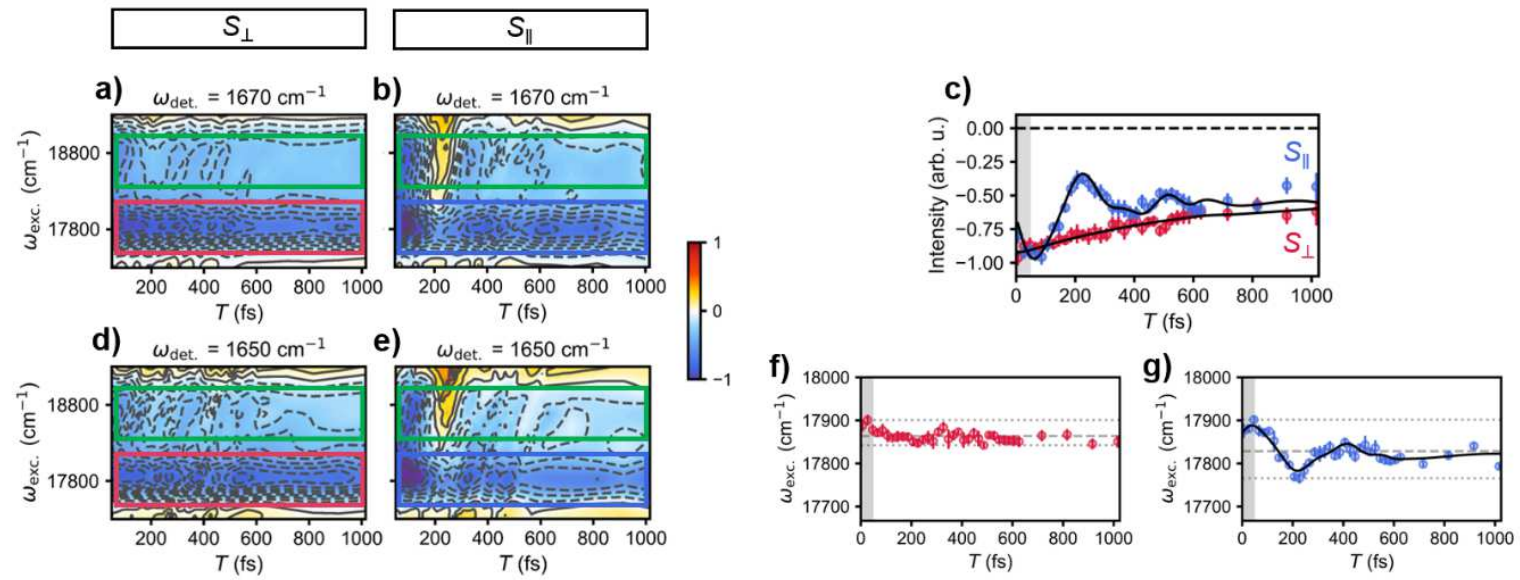

Figure 3. Excited state absorption dynamics along the excitation axis. Perpendicular and parallel PA spectral evolution as a function of waiting time along the excitation axis at a fixed detection frequency of $1670 \mathrm{~cm}^{-1}$. Ground state bleach features are positive (red) features and excited state absorption features are negative (blue) features. The frequency region centered around $17800 \mathrm{~cm}^{-1}$ is boxed in red for the perpendicular PA component $\left(S_{\perp}\right)$ and blue for the parallel PA component $\left(S_{\|}\right)$. Throughout, green boxes indicate the higher frequency region centered around 18700 $\mathrm{cm}^{-1}$. c) Peak amplitude dynamics at a detection frequency of $1650 \mathrm{~cm}^{-1}$ along $17800 \mathrm{~cm}^{-1}$ (through the center of the corresponding boxed regions of the same color). Error bars indicate the estimated errors in the peak amplitude based on the standard deviation of the signal amplitude at each waiting time. The black lines indicate the fits of the peak amplitude dynamics (provided in Supplementary Table 1). The gray shaded region indicates the region where pulse overlap effects occur. d)-e) Same as described for a)-b) except at a detection frequency of $1650 \mathrm{~cm}^{-1}$. f)-g) Evolution of excited state frequency distribution of the perpendicular and parallel PA spectra, respectively, at a detection frequency of $1670 \mathrm{~cm}^{-1}$. The error bars $(1 \sigma$ intervals $)$ indicate the uncertainty in the position of the peak maximum, determined by a Gaussian fit of the peak in the range provided in the y-axis of the plot. The dashed gray line indicates the average center frequency, while dotted gray lines indicate the minimum and maximum frequencies. Again, the gray shaded region indicates the region where pulse overlap effects occur. The black line in $g$ ) indicates the fit of the evolution of the frequency distribution (provided in Supplementary Table 2).

To explain the oscillatory peak dynamics, two main possibilities emerge: 1) rapid vibronic $\mathrm{Q}_{\mathrm{y}}-\mathrm{Q}_{\mathrm{x}} \rightarrow \mathrm{Q}_{\mathrm{y}}$ transfer occurs and is followed by energy transfer between the lower-lying $\mathrm{Q}_{\mathrm{y}}$ states or 2) the oscillatory signal arises from these higher-lying bands themselves. The most straightforward way to verify the origin of this signal is to track the how the optical frequency distribution of this region changes during the waiting time, which is essentially tracking the evolution of the excited state(s) in this region. Dynamical changes in the frequency distribution indicate that the excited state(s) are still populated, which would indicate that the dynamics being observed originate from the higher-lying $\left(\mathrm{Q}_{\mathrm{x}}\right)$ states rather than from the lower-lying $\mathrm{Q}_{\mathrm{y}}$ states. Figure $3 \mathrm{~g}$ presents a plot of how the maximum peak position of the band centered around 17800 $\mathrm{cm}^{-1}$ in $S_{\|}$evolves as a function of waiting time. The peak maximum as a function of waiting time 
was determined via fitting with a Gaussian function. It is evident that the frequency distribution changes dynamically along the waiting time. Actually, a fit of this peak evolution reveals oscillatory frequencies of $92 \pm 6 \mathrm{~cm}^{-1}$ and $240 \pm 20 \mathrm{~cm}^{-1}$ (error indicates $1 \sigma$ interval, see Supplementary Table 2 for complete fit results), which are in agreement within error to those present in the peak amplitude. From the fits, the dominant $92 \pm 6 \mathrm{~cm}^{-1}$ frequency component was found to decay on a timescale of $250 \pm 50 \mathrm{fs}$, similar to the timescales of the same component in

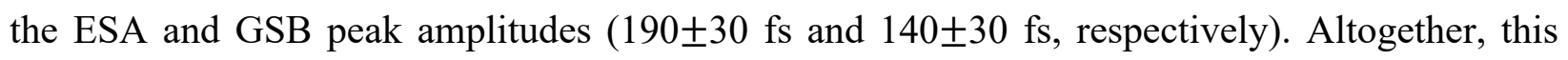
suggests that excitation stays in the $\mathrm{Q}_{\mathrm{x}}$ bands of $\mathrm{Chl} b$ for over $200 \mathrm{fs}$, in agreement with recent theoretical work on $\mathrm{Q}_{\mathrm{x}} \rightarrow \mathrm{Q}_{\mathrm{y}}$ transfer in $\mathrm{Chl} b$, which was found to occur on a timescale of $\sim 200$ fs. ${ }^{36}$ Interestingly, the agreement between these timescales suggests that the $\mathrm{Q}_{\mathrm{x}}$ states of Chl $b$ studied here are not significantly mixed with neighboring pigments, but rather retain significant monomeric-like character (i.e. these observed spectral signatures arise from more localized Chl $b$ pigments). As a control, we show the frequency distribution evolution for the same ESA feature in $S_{\perp}$ (Figure 3f). Clearly, no such oscillatory behavior is observed (just as none was observed in the peak amplitudes for this PA component) or in previous work focused on the lower-lying $\mathrm{Q}_{\mathrm{y}}$ states, ${ }^{19}$ suggesting, again, that the oscillatory behavior is unique to the $\mathrm{Q}_{\mathrm{x}}$ states. Although it remains difficult to assign the exact origin of this signal, especially as the peak evolution analysis of the Chl $b$ GSB feature was hindered due to slightly lower signal, it is likely that these oscillatory signals result from coupling between low frequency chlorin ring distortions and the $\mathrm{Q}_{\mathrm{x}}$ state. Such a phenomenon has been found to occur in other cases for Chls, where the lifting of the electronic TDM out of the ring results in a coupling and subsequent increase in intensity of the chlorin ring distortion modes. ${ }^{40,41}$ The identical, in-phase peak amplitude dynamics between the GSB and ESA of $\mathrm{Chl} b$ character is also particularly notable, and may suggest that the observed dynamics arise 
from non-Condon type coupling. ${ }^{42}$ The presence of such a non-Condon effect may help to facilitate rapid internal conversion, therefore further improving quantum efficiency across the PAR. However, further theoretical efforts are required to support such a conclusion.

\section{Concluding Comments}

This application of PA 2DEV spectroscopy to the highly mixed vibronic $\mathrm{Q}_{\mathrm{y}-\mathrm{Q}} \mathrm{Q}_{\mathrm{x}}$ region of the LHCII spectrum is one of the first major attempts to understand the origin and function of the states that energetically connect the lowest lying Chl $\mathrm{Q}_{\mathrm{y}}$ and Car states - a crucial piece in the complete understanding of the intricate spatial and energetic structure of this photosynthetic antenna complex, as well as the light-harvesting abilities of green plants and algae. With the aid of infrared detection, we show that the states spanning the green portion of the vibronic $Q_{y}-Q_{x}$ region of the LHCII spectrum have significant $\mathrm{Chl} b$ character. More specifically, our results show that the region centered around $17800 \mathrm{~cm}^{-1}$ contains significant contributions from vibronic $\mathrm{Q}_{\mathrm{y}}$ and Qx states of Chl $b$, while the region around $18700 \mathrm{~cm}^{-1}$ has predominately $\mathrm{Chl} b \mathrm{Q}_{\mathrm{x}}$ character. While ultrafast relaxation from the vibronic states of predominately $\mathrm{Chl} b$ character to the lowerlying Qy states occurred within $90 \mathrm{fs}$, a fraction of the excitation was seen to remain in the $\mathrm{Q}_{\mathrm{x}}$ bands of predominately Chl $b$ for over 200 fs (based on an oscillatory feature associated with these states). The agreement between this timescale for Chl $b \mathrm{Q}_{\mathrm{x}} \rightarrow \mathrm{Q}_{\mathrm{y}}$ transfer in LHCII and recent theoretical work on monomeric $\mathrm{Chl} b^{36}$, suggests that the observed $\mathrm{Q}_{\mathrm{x}}$ states arise from more highly localized Chl $b$ pigments. The oscillatory feature likely results from electronic-vibrational coupling between the $\mathrm{Q}_{\mathrm{x}}$ transition and low frequency chlorin ring distortions, although a definitive assignment remains difficult. The addition of polarization control to 2DEV spectroscopy enables the observation of different states in parallel versus perpendicular detection without a change in excitation frequency. This lack of reciprocity between the polarization components enables a new 
way of probing the level structure and dynamics of highly congested and mixed spectra of molecular complexes. These results also highlight the critical role of electronic-nuclear mixing in the extension of efficient light-harvesting across the PAR, as well as a distinct difference between Chl $a$ versus Chl $b$ pigments in light-harvesting. From an in vivo perspective, the significant enhancement in the absorption of these vibronic $Q_{y}-Q_{x}$ states arising from the diffusive nature of the leaf environment (i.e. the detour effect), coupled with the fact that green photons therefore ${ }^{1,24-}$ ${ }^{27}$ penetrate deeper into the mesophyll, indicates that $\mathrm{Chl} b$ is responsible for both harnessing green light and evenly distributing photosynthetic activity throughout the leaf.

\section{Methods}

\section{Sample Preparation}

The isolation of thylakoid membranes was performed by using sucrose cushion $^{43}$ as described below. Deveined leaves were homogenized in $25 \mathrm{mM}$ Tricine-KOH (pH 7.8), $400 \mathrm{mM}$ $\mathrm{NaCl}, 2 \mathrm{mM} \mathrm{MgCl} 2,0.2 \mathrm{mM}$ benzamidine, and $1 \mathrm{mM} \varepsilon$-aminocaproic acid at $4{ }^{\circ} \mathrm{C}$ using a Waring blender for $30 \mathrm{~s}$ with max speed. The homogenate was filtrated through 4 layers of Miracloth, and the filtrate was centrifuged at $27,000 \times g$ for $10 \mathrm{~min}$ at $4{ }^{\circ} \mathrm{C}$. The pellet was resuspended in $25 \mathrm{mM}$ Tricine- $\mathrm{KOH}(\mathrm{pH} 7.8), 150 \mathrm{mM} \mathrm{NaCl}, 5 \mathrm{mM} \mathrm{MgCl}, 0.2 \mathrm{mM}$ benzamidine, and $1 \mathrm{mM} \varepsilon-$ aminocaproic acid. The suspension was loaded on sucrose cushion containing $1.3 \mathrm{M}$ sucrose with $25 \mathrm{mM}$ Tricine- $\mathrm{KOH}$ ( $\mathrm{pH} 7.8$ ), $15 \mathrm{mM} \mathrm{NaCl}$, and $5 \mathrm{mM} \mathrm{MgCl}$, which was overlaid on $1.8 \mathrm{M}$ sucrose with $25 \mathrm{mM}$ Tricine- $\mathrm{KOH}$ (pH 7.8), $15 \mathrm{mM} \mathrm{NaCl}$, and $5 \mathrm{mM} \mathrm{MgCl}_{2}$, and centrifuged at $131,500 \times \mathrm{g}$ for $30 \mathrm{~min}$ at $4{ }^{\circ} \mathrm{C}$ using a SW 32 Ti rotor (Beckman Coulter). Thylakoid membranes sedimented in $1.3 \mathrm{M}$ sucrose cushion were collected and washed with $25 \mathrm{mM}$ Tricine- $\mathrm{KOH}$ (pH 7.8), $15 \mathrm{mM} \mathrm{NaCl}$, and $5 \mathrm{mM} \mathrm{MgCl}$, and centrifuged at $27,000 \times g$ for $15 \mathrm{~min}$ at $4{ }^{\circ} \mathrm{C}$. The pellet was resuspended in $25 \mathrm{mM}$ Tricine- $\mathrm{KOH}$ (pH 7.8), $0.4 \mathrm{M}$ sucrose, $15 \mathrm{mM} \mathrm{NaCl}$, and $5 \mathrm{mM} \mathrm{MgCl}_{2}$, 
and centrifuged at $27,000 \times \mathrm{g}$ for $10 \mathrm{~min}$ at $4{ }^{\circ} \mathrm{C}$. The pellet was resuspended and used as purified thylakoid membranes.

The purified thylakoid membranes were resuspended in $25 \mathrm{mM}$ HEPES-NaOH (pH 7.8) and centrifuged at $15,300 \times \mathrm{g}$ for $10 \mathrm{~min}$ at $4^{\circ} \mathrm{C}$. The pellet was resuspended in $25 \mathrm{mM}$ HEPES$\mathrm{NaOH}(\mathrm{pH} 7.8)$ at $2.0 \mathrm{mg} \mathrm{Chl} / \mathrm{mL}$ and solubilized with $4 \%$ (w/v) n-dodecyl- $\alpha$-D-maltoside ( $\alpha$-DM; Anatrace) for $30 \mathrm{~min}$ with gentle agitation on ice. The unsolubilized membranes were removed by centrifuging at $21,000 \times \mathrm{g}$ for $5 \mathrm{~min}$ at $4{ }^{\circ} \mathrm{C}$. The supernatant was filtrated through $0.22 \mu \mathrm{m}$ filter using Durapore Ultrafree filters centrifuged at $10,000 \times g$ for 3 min at $4{ }^{\circ} \mathrm{C}$. The $200 \mu \mathrm{L}$ of filtered solubilized fraction was used for gel filtration chromatography using the ÄKTAmicro chromatography system with a Superdex 200 Increase 10/300 GL column (GE Healthcare) equilibrated with $25 \mathrm{mM}$ HEPES-NaOH $(\mathrm{pH} 7.8)$ and $0.03 \%(\mathrm{w} / \mathrm{v}) \alpha-\mathrm{DM}$ at room temperature. The flow rate was $0.9 \mathrm{~mL} / \mathrm{min}$. The proteins were detected at $280 \mathrm{~nm}$ absorbance. The fraction separated from 10.0 to $10.3 \mathrm{~mL}$ contained trimeric LHCII proteins.

For the experiments, the maximum optical density of the LHCII sample in the investigated visible range was $\sim 0.25$ with a path length of $200 \mu \mathrm{m}$ at $77 \mathrm{~K}$ (Optistate DN2, Oxford Instruments).

\section{Spectroscopic Measurements}

The 2DEV experimental setup used in this work, which has been described in detail elsewhere ${ }^{30}$, will be outlined briefly below. A home-built visible NOPA and mid-IR OPA were pumped by a Ti:Sapphire oscillator (Vitara-S, Coherent) and regenerative amplifier (Legend Elite, Coherent). The NOPA was tuned such that the center frequency of the visible pump pulse spectrum was set to $\sim 18415 \mathrm{~cm}^{-1}$ and spanned $17545 \sim 19230 \mathrm{~cm}^{-1}$. A prism pair in combination with a pulse shaper (Dazzler, Fastlite) were used to compress the visible pulses ( $\sim 35 \mathrm{fs})$. The energy of the visible pump pulses was $\sim 160 \mathrm{~nJ}$ and a $f=25 \mathrm{~cm}$ silver coated $90^{\circ}$ off-axis parabolic mirror was 
employed to focus the visible pulses into the sample to a spot size of $250 \mu \mathrm{m}$. The mid-IR OPA was tuned to produce an IR probe spectrum with a center frequency of $\sim 1620 \mathrm{~cm}^{-1}$. The mid-IR pulse was split by a 50:50 ZnSe beam splitter, forming probe and reference beams, where the probe was normalized by the reference, in order to account for shot-to-shot energy fluctuations in the IR source. The IR probe and reference pulses both had an energy of $\sim 100 \mathrm{~nJ}$ and duration of $\sim 60 \mathrm{fs}$. Both IR pulses were focused into the sample to a spot size of $200 \mu \mathrm{m}$ with a $f=15 \mathrm{~cm}$ gold coated $90^{\circ}$ off-axis parabolic mirror. After the sample, the IR probe and reference were dispersed with a spectrometer (Triax 180, Horiba) onto a dual-array 64 element HgCdTe detector (Infrared Systems Development).

As the 2DEV experiments were performed in a partially collinear pump-probe geometry, the pulse shaper was also employed to generate the visible pump pulse pair and to control the relative phase (where the $2 \mathrm{DEV}$ signal was isolated with a $3 \times 1$ phase cycling scheme) (4, $^{45}$ and initial time delay, $t_{1}$, (scanned from $0-100 \mathrm{fs}$ in $\sim 2.4 \mathrm{fs}$ steps) between the pulses. To remove the optical frequency of the pump, the data was collected in the fully rotated frame with respect to $t_{1}$. The visible pulse pair was directed towards the sample via a retroreflector on a motorized delay stage used to control the waiting time, $T$, between the pump pair and probe pulses. In this work, polarization-dependent $2 \mathrm{DEV}$ spectra were collected as a function of waiting time in 10 fs increments from 0 fs to $625 \mathrm{fs}$ and in $100 \mathrm{fs}$ increments from 715 fs to $1015 \mathrm{fs}$. The relative polarization between the pump pulses and the probe pulse was controlled with a $\lambda / 2$ waveplate in the pump beam line.

Except for the NOPA, the entire setup was purged with dry air, free of $\mathrm{CO}_{2}($ Perkins Balston FT-IR Purge Gas Generator). 


\section{Data Processing}

For correct visualization, all data presented in this work have been adjusted for a small residual positive chirp of $\sim 0.1 \frac{\mathrm{fs}}{\mathrm{cm}^{-1}}$ in the visible pump pulses, which were unable to be fully compressed to the transform limit by the pulse shaper and prism pair.

\section{Data Availability}

The data presented in this study are available from the corresponding author upon reasonable request.

\section{References}

1. Terashima, I., Fujita, T., Inoue, T., Chow, W. S. \& Oguchi, R. Green light drives leaf photosynthesis more efficiently than red light in strong white light: Revisiting the enigmatic question of why leaves are green. Plant Cell Physiol. 50, 684-697 (2009).

2. Blankenship, R. E. Molecular Mechanisms of Photosynthesis. (Blackwell Science Ltd, 2002).

3. Liu, Z. et al. Crystal structure of spinach major light-harvesting complex at $2.72 \AA$ resolution. Nature 428, 287-292 (2004).

4. Blankenship, R. E. Early Evolution of Photosynthesis. Plant Physiol. 154, 434-438 (2010).

5. Buick, R. When did oxygenic photosynthesis evolve? Philos. Trans. R. Soc. B Biol. Sci. 363, 2731-2743 (2008).

6. Lewis, N. H. C. et al. Observation of electronic excitation transfer through light harvesting complex II using two-dimensional electronic-vibrational spectroscopy. J. Phys. Chem. Lett. 
7, 4197-4206 (2016).

7. Connelly, J. P. et al. Ultrafast spectroscopy of trimeric light-harvesting complex II from higher plants. J. Phys. Chem. B 101, 1902-1909 (1997).

8. Wells, K. L., Lambrev, P. H., Zhang, Z., Garab, G. \& Tan, H. S. Pathways of energy transfer in LHCII revealed by room-temperature 2D electronic spectroscopy. Phys. Chem. Chem. Phys. 16, 11640-11646 (2014).

9. Schlau-Cohen, G. S. et al. Pathways of energy flow in LHCII from two-dimensional electronic spectroscopy. J. Phys. Chem. B 113, 15352-15363 (2009).

10. Calhoun, T. R. et al. Quantum coherence enabled determination of the energy landscape in light-harvesting complex II. J. Phys. Chem. B 113, 16291-16295 (2009).

11. Schlau-Cohen, G. S. et al. Spectroscopic elucidation of uncoupled transition energies in the major photosynthetic light-harvesting complex, LHCII. Proc. Natl. Acad. Sci. 107, 13276$13281(2010)$.

12. Schlau-Cohen, G. S. et al. Elucidation of the timescales and origins of quantum electronic coherence in LHCII. Nat. Chem. 4, 389-395 (2012).

13. Agarwal, R. et al. Ultrafast Energy Transfer in LHC-II Revealed by Three-Pulse Photon Echo Peak Shift Measurements. J. Phys. Chem. B 104, 2908-2918 (2000).

14. Novoderezhkin, V. I., Palacios, M. A., Van Amerongen, H. \& Van Grondelle, R. Energytransfer dynamics in the LHCII complex of higher plants: modified Redfield approach. $J$. Phys. Chem. B 108, 10363-10375 (2004).

15. Novoderezhkin, V. I., Palacios, M. A., Van Amerongen, H. \& Van Grondelle, R. Excitation 
dynamics in the LHCII complex of higher plants: modeling based on the $2.72 \AA$ crystal structure. J. Phys. Chem. B 109, 10493-10504 (2005).

16. Novoderezhkin, V., Marin, A. \& Van Grondelle, R. Intra- and inter-monomeric transfers in the light harvesting LHCII complex: The Redfield-Förster picture. Phys. Chem. Chem. Phys. 13, 17093-17103 (2011).

17. Son, M., Pinnola, A., Bassi, R. \& Schlau-Cohen, G. S. The Electronic Structure of Lutein 2 Is Optimized for Light Harvesting in Plants The Electronic Structure of Lutein 2 Is Optimized for Light Harvesting in Plants. Chem 5, 575-584 (2019).

18. Son, M., Pinnola, A., Gordon, S. C., Bassi, R. \& Schlau-Cohen, G. S. Observation of dissipative chlorophyll-to-carotenoid energy transfer in light-harvesting complex II in membrane nanodiscs. Nat. Commun. 11, 1295 (2020).

19. Arsenault, E. A., Yoneda, Y., Iwai, M., Niyogi, K. K. \& Fleming, G. R. Vibronic mixing enables ultrafast energy flow in light-harvesting complex II. Nat. Commun. 11, 1-8 (2020).

20. Duan, H. G. et al. Two-dimensional electronic spectroscopy of light-harvesting complex II at ambient temperature: a joint experimental and theoretical study. J. Phys. Chem. B 119, 12017-12027 (2015).

21. Du, M., Xie, X., Mets, L. \& Fleming, G. R. Direct observation of ultrafast energy-transfer processes in light harvesting complex II. J. Phys. Chem. 98, 4736-4741 (1994).

22. Zhang, Z., Lambrev, P. H., Wells, K. L., Garab, G. \& Tan, H. S. Direct observation of multistep energy transfer in LHCII with fifth-order 3D electronic spectroscopy. Nat. Commun. 6, 1-7 (2015). 
23. Polívka, T. et al. Carotenoid S1 state in a recombinant light-harvesting complex of photosystem II. Biochemistry 41, 439-450 (2002).

24. Rivadossi, A., Zucchelli, G., Garlaschi, F. M. \& Jennings, R. C. Light Absorption by the Chlorophyll a-b Complexes of Photosystem II in a Leaf with Special Reference to LHCII. Photochem. Photobiol. 80, 492-498 (2004).

25. Garlaschi, F. M., Zucchelli, G. \& Jennings, R. C. Studies on light absorption and photochemical activity changes in chloroplast suspensions and leaves due to light scattering and light filtration across chloroplast and vegetation layers. Photosynth. Res. 20, 207-220 (1989).

26. Kume, A. Importance of the green color, absorption gradient, and spectral absorption of chloroplasts for the radiative energy balance of leaves. J. Plant Res. 130, 501-514 (2017).

27. Smith, H. L., Mcausland, L. \& Murchie, E. H. Don't ignore the green light: Exploring diverse roles in plant processes. J. Exp. Bot. 68, 2099-2110 (2017).

28. Blankenship, R. E. et al. Comparing photosynthetic and photovoltaic efficiencies and recognizing the potential for improvement. Science (80-. ). 332, 805-809 (2011).

29. Zhu, X. G., Long, S. P. \& Ort, D. R. What is the maximum efficiency with which photosynthesis can convert solar energy into biomass? Curr. Opin. Biotechnol. 19, 153-159 (2008).

30. Oliver, T. A. A., Lewis, N. H. C. \& Fleming, G. R. Correlating the motion of electrons and nuclei with two-dimensional electronic-vibrational spectroscopy. Proc. Natl. Acad. Sci. 111, 10061-10066 (2014). 
31. Gaynor, J. D., Sandwisch, J. \& Khalil, M. Vibronic coherence evolution in multidimensional ultrafast photochemical processes. Nat. Commun. 10, 5621 (2019).

32. Wu, E. C. et al. Two-dimensional electronic-vibrational spectroscopic study of conical intersection dynamics: An experimental and electronic structure study. Phys. Chem. Chem. Phys. 21, 14153 (2019).

33. Roy, P. P. et al. Solvent Mediated Excited State Proton Transfer in Indigo Carmine. J. Phys. Chem. Lett. 11, 4156-4162 (2020).

34. Gaynor, J. D., Petrone, A., Li, X. \& Khalil, M. Mapping vibronic couplings in a solar cell dye with polarization-selective two-dimensional electronic-vibrational spectroscopy. $J$. Phys. Chem. Lett. 9, 6289-6295 (2018).

35. Lewis, N. H. C. \& Fleming, G. R. Two-dimensional electronic-vibrational spectroscopy of chlorophyll a and b. J. Phys. Chem. Lett. 7, 831-837 (2016).

36. Bricker, W. P. et al. Non-radiative relaxation of photoexcited chlorophylls: Theoretical and experimental study. Sci. Rep. 5, 1-16 (2015).

37. Thyrhaug, E., Žídek, K., Dostál, J., Bína, D. \& Zigmantas, D. Exciton Structure and Energy Transfer in the Fenna-Matthews-Olson Complex. J. Phys. Chem. Lett. 7, 1653-1660 (2016).

38. Read, E. L. et al. Cross-peak-specific two-dimensional electronic spectroscopy. Proc. Natl. Acad. Sci. 104, 14203-14208 (2007).

39. Houssier, C. \& Sauer, K. Circular Dichroism and Magnetic Circular Dichroism of the Chlorophyll and Protochlorophyll Pigments. J. Am. Chem. Soc. 92, 779-791 (1970).

40. Diers, J. R., Zhu, Y., Blankenship, R. E. \& Bocian, D. F. Qy-excitation resonance Raman 
spectra of chlorophyll a and bacteriochlorophyll c/d aggregates. Effects of peripheral substituents on the low-frequency vibrational characteristics. J. Phys. Chem. 100, 85738579 (1996).

41. Zhou, C., Diers, J. R. \& Bocian, D. F. Qy-excitation resonance raman spectra of chlorophyll a and related complexes. Normal mode characteristics of the low-frequency vibrations. $J$. Phys. Chem. B 101, 9635-9644 (1997).

42. Kano, H., Saito, T. \& Kobayashi, T. Observation of Herzberg-Teller-type wave packet motion in porphyrin J-aggregates studied by Sub-5-fs spectroscopy. J. Phys. Chem. A 106, $3445-3453$ (2002).

43. Iwai, M. et al. Light-harvesting complex Lhcb9 confers a green alga-type photosystem i supercomplex to the moss Physcomitrella patens. Nat. Plants 1, 14008 (2015).

44. Myers, J. A., Lewis, K. L., Tekavec, P. F. \& Ogilvie, J. P. Two-color two-dimensional Fourier transform electronic spectroscopy with a pulse-shaper. Opt. Express 16, 17420 (2008).

45. Zhang, Z., Wells, K. L., Hyland, E. W. J. \& Tan, H. S. Phase-cycling schemes for pumpprobe beam geometry two-dimensional electronic spectroscopy. Chem. Phys. Lett. 550, 156-161 (2012).

\section{Acknowledgements}

This research was supported by the U.S. Department of Energy, Office of Science, Basic Energy Sciences, Chemical Sciences, Geosciences, and Biosciences Division. E.A.A. acknowledges the support of the National Science Foundation Graduate Research Fellowship 
(Grant No. DGE 1752814). Y.Y. appreciates the support of the Japan Society for the Promotion of Science (JSPS) Postdoctoral Fellowship for Research Abroad.

\section{Author Contributions}

E.A.A. and G.R.F. conceived the research. E.A.A. and Y.Y. performed the 2DEV experiments. E.A.A. analyzed the experimental data. E.A.A., Y.Y., and G.R.F. discussed the experimental results. M.I. prepared the sample. E.A.A. and G.R.F. wrote the paper. E.A.A, Y.Y, M.I, K.K.N., and G.R.F. commented on the manuscript.

\section{Competing Interests}

The authors declare no competing interests. 


\section{Figures}

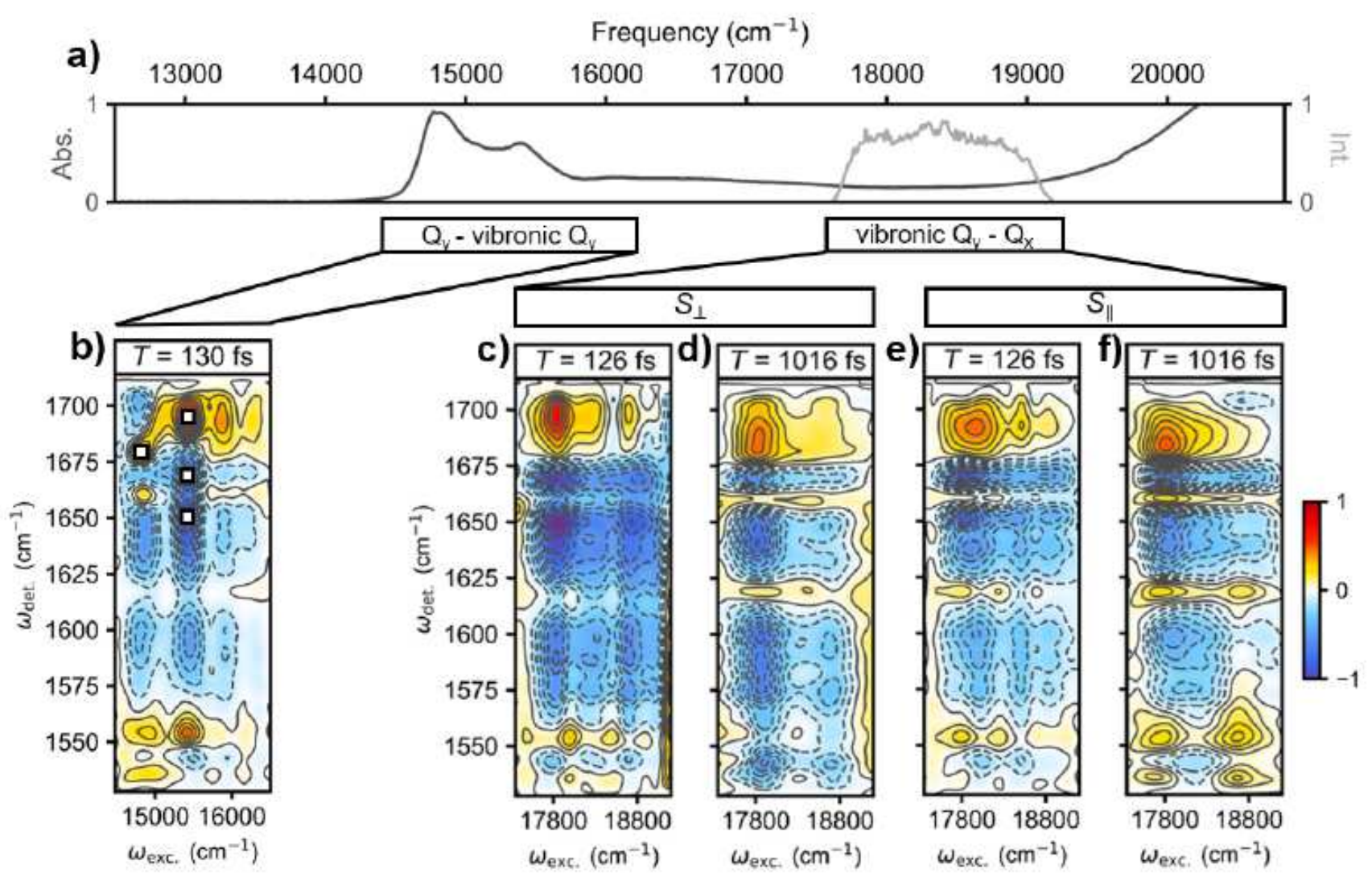

Figure 1

Linear absorption and two-dimensional electronic-vibrational spectra of LHCII. a) Linear absorption spectrum of LHCll at $77 \mathrm{~K}$, along with the spectrum of the visible pump pulses employed in this work (light gray). b) Representative 2DEV spectrum of the Qy band of LHCll at T = $130 \mathrm{fs}$, prior to significant $\mathrm{Chl} b \otimes \mathrm{Chl}$ a transfer. Throughout, ground state bleach (GSB) features are positive (red) features and excited state absorption (ESA) features are negative (blue) features. Four regions have been marked in the spectra with white squares - one GSB in the predominately $\mathrm{Chl}$ a excitonic band (centered around $14800 \mathrm{~cm}-1$ ) and one GSB and two ESA in the predominately $\mathrm{Chl} b$ excitonic band (centered around $15500 \mathrm{~cm}-1$ ). 2c)-d) Perpendicular PA 2DEV spectra of the vibronic Qy-Qx bands of LHCIl at $77 \mathrm{~K}$ at waiting times of $T=126 \mathrm{fs}$ and $1016 \mathrm{fs}$. e)-f) Parallel PA 2DEV spectra of the vibronic Qy-Qx bands of $\mathrm{LHCll}$ at $77 \mathrm{~K}$ at waiting times of $\mathrm{T}=126 \mathrm{fs}$ and $1016 \mathrm{fs}$. 

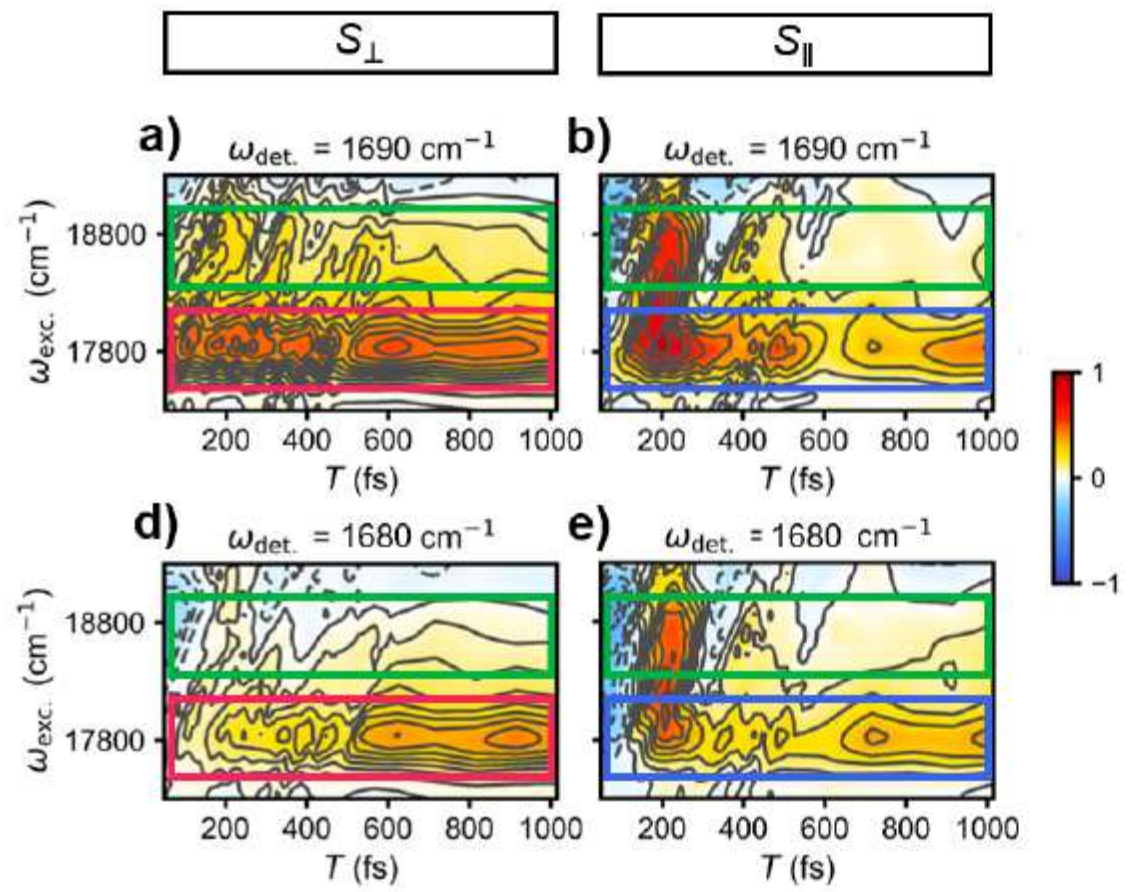

c)
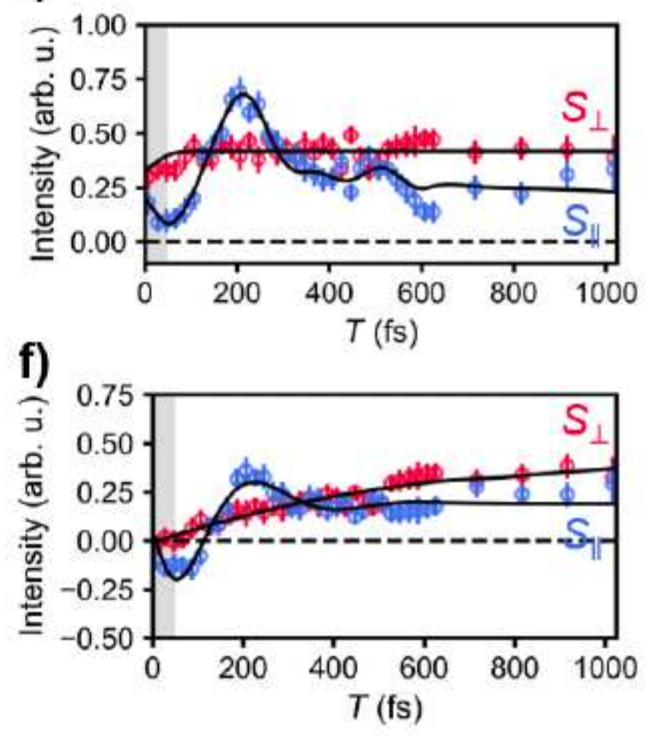

\section{Figure 2}

Ground state bleach dynamics along the excitation axis. a)-b) Perpendicular and parallel PA spectral evolution as a function of waiting time along the excitation axis at a fixed detection frequency of 1690 $\mathrm{cm}-1$ (indicative of $\mathrm{Chl} b$ population). Ground state bleach features are positive (red) features and excited state absorption features are negative (blue) features. The frequency region centered around $17800 \mathrm{~cm}-1$ is boxed in red for the perpendicular PA component (S区) and blue for the parallel PA component (S区). Throughout, green boxes indicate the higher frequency region centered around $18700 \mathrm{~cm}-1$. c) Peak amplitude dynamics at a detection frequency of $1690 \mathrm{~cm}-1$ along $17800 \mathrm{~cm}-1$ (through the center of the corresponding boxed regions of the same color). Error bars indicate the estimated errors in the peak amplitude based on the standard deviation of the signal amplitude at each waiting time. The black lines indicate the fits of the peak amplitude dynamics (provided in Supplementary Table 1). The gray shaded region indicates the region where pulse overlap effects occur. d)-e) Same as described for a)-b) except at a detection frequency of $1680 \mathrm{~cm}-1$ (indicative of $\mathrm{Chl}$ a population). $\mathrm{f}$ ) Same as c) except at a detection frequency of $1680 \mathrm{~cm}-1$. 

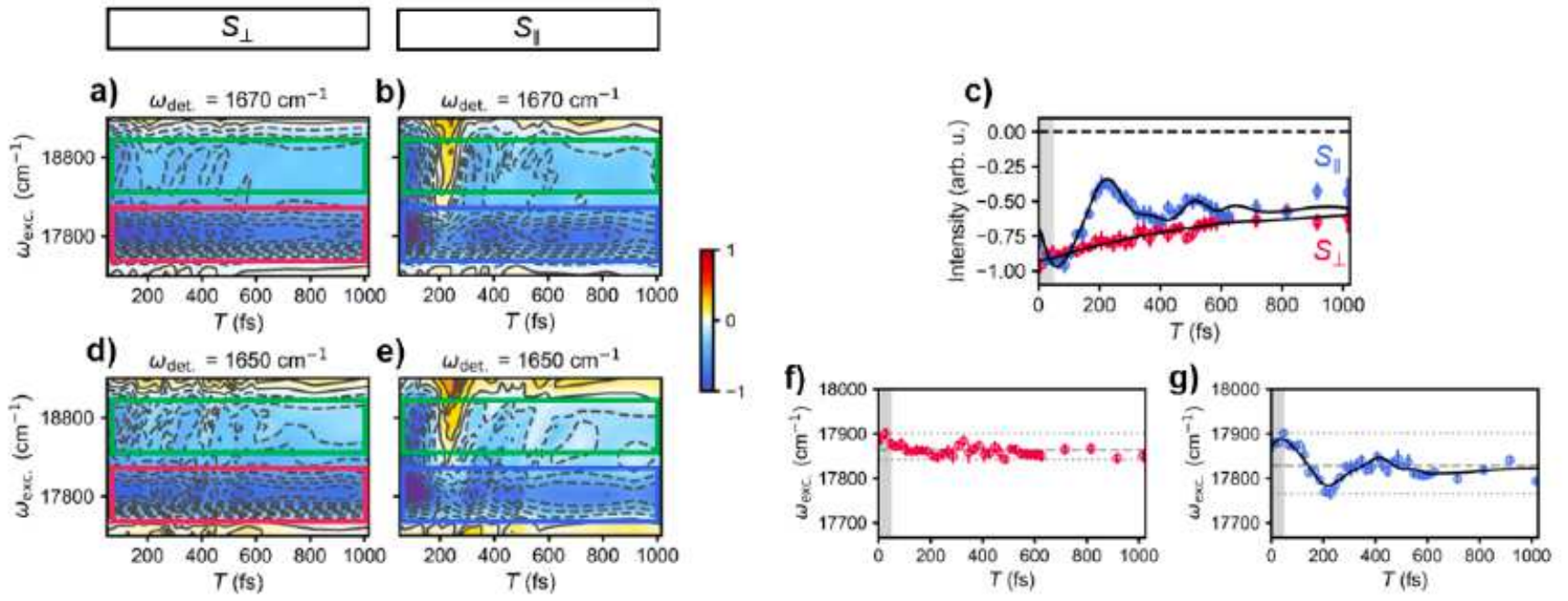

\section{Figure 3}

Excited state absorption dynamics along the excitation axis. Perpendicular and parallel PA spectral evolution as a function of waiting time along the excitation axis at a fixed detection frequency of 1670 $\mathrm{cm}-1$. Ground state bleach features are positive (red) features and excited state absorption features are negative (blue) features. The frequency region centered around $17800 \mathrm{~cm}-1$ is boxed in red for the perpendicular PA component (S区) and blue for the parallel PA component (S区). Throughout, green boxes indicate the higher frequency region centered around $18700 \mathrm{~cm}-1$. c) Peak amplitude dynamics at a detection frequency of $1650 \mathrm{~cm}-1$ along $17800 \mathrm{~cm}-1$ (through the center of the corresponding boxed regions of the same color). Error bars indicate the estimated errors in the peak amplitude based on the standard deviation of the signal amplitude at each waiting time. The black lines indicate the fits of the peak amplitude dynamics (provided in Supplementary Table 1). The gray shaded region indicates the region where pulse overlap effects occur. d)-e) Same as described for a)-b) except at a detection frequency of $1650 \mathrm{~cm}-1 . \mathrm{f})$-g) Evolution of excited state frequency distribution of the perpendicular and parallel PA spectra, respectively, at a detection frequency of $1670 \mathrm{~cm}-1$. The error bars (1区 intervals) indicate the uncertainty in the position of the peak maximum, determined by a Gaussian fit of the peak in the range provided in the $y$-axis of the plot. The dashed gray line indicates the average center frequency, while dotted gray lines indicate the minimum and maximum frequencies. Again, the gray shaded region indicates the region where pulse overlap effects occur. The black line in g) indicates the fit of the evolution of the frequency distribution (provided in Supplementary Table 2).

\section{Supplementary Files}

This is a list of supplementary files associated with this preprint. Click to download. 